\title{
Quantized Density Response in Insulators
}

\author{
Qian Niu
}

Department of Physics, University of Texas, Austin, Texas 78712

\begin{abstract}
The response of particle density to a dilation of a periodic potential in an insulator, with or without a fixed background potential or a magnetic field, is shown to be quantized. A similar phenomenon occurs in a quantum Hall system, where the derivative of the electron density with respect to the magnetic field is quantized in units of $e / h$, the inverse flux quantum. A number of other interesting results may be unified under the notion of quantized density response, including the quantization of adiabatic particle transport, the gap labeling theorem and its generalization in the presence of a magnetic field.
\end{abstract}

PACS: $02.40 .+\mathrm{m}, 72.15 . \mathrm{Gd}$ 


\section{Introduction}

It is well known that for a two dimensional gas of electrons in a magnetic field $B$, the particle density $\rho$ satisfies $\rho /(e B / h)=n$ for $n$ filled Landau levels below the Fermi energy, where $h / e$ is the flux quantum. In the presence of a weak periodic potential, each

Landau level is split into $p$ subbands, if the magnetic flux in each unit cell is $\frac{p}{q}$ times the flux quantum, where $q$ and $p$ are mutually prime integers. When the Fermi energy lies in a gap between two such subbands, the ratio $\rho /(e B / h)$ is no longer an integer, because each filled subband contains a particle density $p$ times smaller than that for a full Landau level. What remains to be quantized is instead the derivative

$$
\frac{\partial \rho}{\partial(e B / h)},
$$

which is also the integer for the quantized Hall conductance.[1-4] Indeed, according to the Streda formula,[1] the Hall conductance may be generally written as

$$
e\left(\frac{\partial \rho}{\partial B}\right)_{\mu}
$$

whenever the Fermi energy $\mu$ lies in an energy gap (or even a mobility gap). Under the same condition, the Hall conductance and therefore the density response are quantized. Note that disorder is certainly allowed under this condition.

In this paper, we would like to show that the notion of quantized density response may be applied to other type of insulators. Consider now an electron gas in a periodic potential. The average particle density satisfies $\rho v=n$ for $n$ filled Bloch bands, where $v$ is the unit cell volume. A natural question is whether one can generalize such a result to the case with an additional background potential and/or a magnetic field. The background potential may be periodic with a different periodicity or even be disordered. The magnetic field does not have to be uniform. In analogy with the quantum Hall system discussed above, we would like to expect that the density response to a dilation of a periodic potential should be quantized when the Fermi energy lies in a gap (or even a mobility gap), although the density itself may not be quantized. We show in this paper that this is indeed the case for a number of interesting situations. We would like to emphasize that the notion of quantized density response may provide a unifying framework in which several important 
subjects, such as the quantum Hall effect, quantized adiabatic particle transport, and gap labeling theorem for quasi-periodic systems, are found as applications.

The organization of our paper is as follows. Our initial study will be limited to the relatively simple case of one dimensional systems (Section 2), but the result will be generalized to two dimensions afterwards (Section 3). The presence of particle-particle interactions makes the problem complicated, but does not alter our conclusion at least for one dimensional systems (Section 4). Density response to a magnetic field will then be studied in Section 5. Some useful applications of our results will be discussed in Section 6 .

\section{One Dimensional Case}

Consider a system of non-interacting electrons in the following potential at zero temperature:

$$
V(x)=V_{1}(\alpha x-\xi)+V_{2}(x)
$$

where $V_{1}$ is a periodic function of $\xi$ of period 1 , so that the periodicity in $x$ is $1 / \alpha$. We will assume that the Fermi energy lies in an energy gap for arbitrary $\xi$ and for a small

neighborhood of $\alpha$. We would like to show that $\frac{\partial \bar{\rho}}{\partial \alpha}$ is quantized as integers, where $\bar{\rho}$ is the density averaged over position in the bulk of the system and over $\xi$ in a period (unity). The average over $\xi$ will be convenient for the mathematical manipulations, but is not really necessary if the potential $V_{2}$ is random, in which case there is no prefered position for $V_{1}$. The same is also true when $V_{2}$ is periodic but incommensurate with $V_{1}$.

The particle density at $x_{0}$ may be written as

$$
\rho\left(x_{0}\right)=\oint \frac{d z}{2 \pi i} \operatorname{Tr}\left\{G \hat{\rho}\left(x_{0}\right)\right\}
$$

where $G=(z-H)^{-1}$ is the resolvent of the single particle Hamiltonian, and $\hat{\rho}\left(x_{0}\right)=$ $\delta\left(x-x_{0}\right)$ is the density operator. The integral goes along a contour in the complex energy plane enclosing the spectrum of filled states. The contour will be chosen everywhere away from the real axis where the spectrum lies, except at the Fermi energy and a point below the whole spectrum. We assume that there are no extended states in the bulk at the Fermi energy. It then follows that the Green function in the coordinate representation $G\left(x, x^{\prime}\right)$ 
is essentially local, i,e., it decays exponentially at large $\left|x-x^{\prime}\right|$.[5] Our later manipulations will depend critically on this property.

Using the differential formula $d G=-G d G^{-1} G$, the density response can be written as

$$
\frac{\partial \rho\left(x_{0}\right)}{\partial \alpha}=-\oint \frac{d z}{2 \pi i} \operatorname{Tr}\left\{G \frac{\partial V_{1}(\alpha x-\xi)}{\partial \xi} x G \hat{\rho}\left(x_{0}\right)\right\} .
$$

We have expressed the $\alpha$-derivative of $V_{1}$ in terms of its $\xi$-derivative. Next, we note that $x G$ may be written as $G\left[G^{-1}, x\right] G+G x=G i \hbar v G+G x$, where $v$ is the velocity operator. Using further the fact that $\frac{\partial V_{1}(\alpha x-\xi)}{\partial \xi}=-\frac{\partial G^{-1}}{\partial \xi}$, we may write (5) as

$$
\frac{\partial \rho\left(x_{0}\right)}{\partial \alpha}=-\oint \frac{d z}{2 \pi i} \operatorname{Tr}\left\{G \frac{\partial V_{1}(\alpha x-\xi)}{\partial \xi} G i \hbar v G \hat{\rho}\left(x_{0}\right)\right\}+\frac{\partial}{\partial \xi} \oint \frac{d z}{2 \pi i} \operatorname{Tr}\left\{G x \hat{\rho}\left(x_{0}\right)\right\} .
$$

When we average over $\xi$ in a unit cell, the second term on the right hand side of (6) becomes zero because of the periodicity of $G$ in $\xi$. In the following, we will no longer consider this term.

The locality of the Green function allows us to do a number of things. First, the unboundedness of $x$ should not introduce any difficulty in the above manipulations, if we measure the density only in the bulk of the system. Secondly, the locality of $G$ makes sure that the expression (6) is insensitive to changes in the boundary condition. In particular, we may impose the quasi-periodic boundary condition $\psi(x+L)=e^{i \theta} \psi(x)$, and make an average over $\theta$. Under such a boundary condition, the average of $\rho\left(x_{0}\right)$ over $x_{0}$ in the bulk may be achieved by integrating over $x_{0}$ in the whole space and dividing it by $L$. Therefore, with $\rho$ also averaged over $\xi$, we have

$$
\frac{\partial \bar{\rho}}{\partial \alpha}=-\frac{1}{L} \int_{0}^{2 \pi} \frac{d \theta}{2 \pi} \int_{0}^{1} d \xi \oint \frac{d z}{2 \pi i} \operatorname{Tr}\left\{G \frac{\partial V_{1}(\alpha x-\xi)}{\partial \xi} G i \hbar v G\right\} .
$$

To show explicitly the quantization of the density response, we make a gauge transformation $\psi(x) \rightarrow \tilde{\psi}(x)=\psi(x) e^{-i \theta x / L}$, then $G \rightarrow \tilde{G}$, and $v \rightarrow-\frac{L}{\hbar} \frac{\partial \tilde{G}^{-1}}{\partial \theta}$, and the expression (7) may be written as

$$
\begin{aligned}
& -\int_{0}^{2 \pi} \frac{d \theta}{2 \pi} \int d \xi \oint \frac{d z}{2 \pi} \operatorname{Tr}\left\{\tilde{G} \frac{\partial \tilde{G}^{-1}}{\partial \xi} \tilde{G} \frac{\partial \tilde{G}^{-1}}{\partial \theta} \tilde{G}\right\} \\
= & \int_{0}^{2 \pi} \frac{d \theta}{2 \pi} \int d \xi \oint \frac{d z}{2 \pi} \operatorname{Tr}\left\{\tilde{G} \frac{\partial \tilde{G}^{-1}}{\partial \theta} \tilde{G} \frac{\partial \tilde{G}^{-1}}{\partial \xi} \tilde{G}\right\},
\end{aligned}
$$


where the second line is derived from the first by using the fact that $\tilde{G} \tilde{G}=-\frac{\partial \tilde{G}}{\partial z}$ and by integrating over $z$ by parts. According to Ref.[6], the above integral takes integer values and represents a topological invariant for mappings from $T^{3}$ to nonsingular operators.

Finally, we would like to remark that the condition that the Green function falls off exponentially at large distances is sufficient but not necessary. A sufficiently fast power law fall off (e.g. $\left|x-x^{\prime}\right|^{-3}$ in 1D) should still be alright for the quantization of density response in the thermaodynamic limit. Such a remark also applies to situations to be discussed in the subsequent sections.

\section{Two Dimensional Case}

The generalization of our result to higher dimensions involves surprisingly a lot more algebra. We have so far only achieved this for two dimenions. The potential $V_{1}$ in $(3)$ now has the form $V_{1}\left(\alpha_{1} x_{1}-\xi_{1}, \alpha_{2} x_{2}-\xi_{2}\right)$, which is periodic in $\xi_{1}$ and $\xi_{2}$ with unit periodicity. We would like to show that

$$
\frac{\partial^{2} \bar{\rho}}{\partial \alpha_{1} \partial \alpha_{2}}
$$

is quantized as integers, where $\bar{\rho}$ is the density averaged over the position in the bulk of the system and over $\xi_{1}$ and $\xi_{2}$. Again, the average over the $\xi$ 's is not really necessary if $V_{2}$ is random or incommensurate with $V_{1}$. We will also assume that there are no extended states in the bulk at the Fermi energy, but states at the boundary, extended or not, are allowed. The system may also be in a magnetic field, which may or may not be uniform. The locality of the Green function will again be the key to our problem.

The general method is quite parallel to the one dimensional case. Equation (5) now becomes

$$
\begin{array}{r}
\frac{\partial^{2} \rho\left(\mathbf{r}_{0}\right)}{\partial \alpha_{1} \partial \alpha_{2}}=\oint \frac{d z}{2 \pi i} \operatorname{Tr}\left\{\hat { \rho } ( \mathbf { r } _ { 0 } ) \left[G \frac{\partial V_{1}}{\partial \xi_{1}} x_{1} G \frac{\partial V_{1}}{\partial \xi_{2}} x_{2} G\right.\right. \\
+G \frac{\partial V_{1}}{\partial \xi_{2}} x_{2} G \frac{\partial V_{1}}{\partial \xi_{1}} x_{1} G \\
\left.\left.+G \frac{\partial^{2} V_{1}}{\partial \xi_{1} \partial \xi_{2}} x_{1} x_{2} G\right]\right\} .
\end{array}
$$

The term including the double derivative of $V_{1}$ may be rewritten as

$$
-G \frac{\partial V_{1}}{\partial \xi_{1}} G x_{1} x_{2} \frac{\partial V_{1}}{\partial \xi_{2}} G-G x_{1} x_{2} \frac{\partial V_{1}}{\partial \xi_{2}} G \frac{\partial V_{1}}{\partial \xi_{1}} G
$$


where we have ignored a term of total derivative in $\xi_{1}$, because such a term will become zero when we average over $\xi_{1}$ in (9). The quantity in the square bracket in (10) may now be written as

$$
\begin{aligned}
& G \frac{\partial V_{1}}{\partial \xi_{1}}\left[x_{1}, G\right] \frac{\partial V_{1}}{\partial \xi_{2}} x_{2} G+G x_{2} \frac{\partial V_{1}}{\partial \xi_{2}}\left[G, x_{1}\right] \frac{\partial V_{1}}{\partial \xi_{1}} G \\
= & G \frac{\partial V_{1}}{\partial \xi_{1}} G i \hbar v_{1} G \frac{\partial V_{1}}{\partial \xi_{2}} x_{2} G-G x_{2} \frac{\partial V_{1}}{\partial \xi_{2}} G i \hbar v_{1} G \frac{\partial V_{1}}{\partial \xi_{1}} G,
\end{aligned}
$$

where we have used the fact that $\left[x_{1}, G\right]=G\left[G^{-1}, x_{1}\right] G=G i \hbar v_{1} G$. Next, we may replace $x_{2} G$ in the first term and $G x_{2}$ in the second term of the last expression by $\pm\left[x_{2}, G\right]=$ $\pm G i \hbar v_{2} G$ respectively, because the error made in (10) is proportional to a total derivative in $\xi_{1}$ and $\xi_{2}$ :

$$
\begin{aligned}
& \operatorname{Tr}\left\{\hat{\rho}\left(\mathbf{r}_{0}\right)\left[G \frac{\partial V_{1}}{\partial \xi_{1}} G v_{1} G \frac{\partial V_{1}}{\partial \xi_{2}} G x_{2}-x_{2} G \frac{\partial V_{1}}{\partial \xi_{2}} G v_{2} G \frac{\partial V_{1}}{\partial \xi_{1}} G\right]\right\} \\
= & \operatorname{Tr}\left\{\hat{\rho}\left(\mathbf{r}_{0}\right) x_{2}\left[\frac{\partial}{\partial \xi_{1}}\left(G v_{1} \frac{\partial G}{\partial \xi_{2}}\right)-\frac{\partial}{\partial \xi_{2}}\left(G v_{1} \frac{\partial G}{\partial \xi_{1}}\right)\right]\right\},
\end{aligned}
$$

where we have used the cyclic property of the trace and the commutativity of $x_{2}$ and $\hat{\rho}\left(\mathbf{r}_{0}\right)$. As a result of these considerations, we may now rewrite the quantity in (10) averaged over $\xi_{1}$ and $\xi_{2}$ as

$$
\iint d \xi_{1} d \xi_{2} \oint \frac{d z}{2 \pi i} \operatorname{Tr}\left\{\hat{\rho}\left(\mathbf{r}_{0}\right)\left[G \frac{\partial V_{1}}{\partial \xi_{1}} G i \hbar v_{1} G \frac{\partial V_{1}}{\partial \xi_{2}} G i \hbar v_{2} G+G i \hbar v_{2} G \frac{\partial V_{1}}{\partial \xi_{2}} G i \hbar v_{1} G \frac{\partial V_{1}}{\partial \xi_{1}} G\right]\right\}
$$

Finally, as in the one dimensional case, we introduce the quasiperiodic boundary conditions in both $x$ and $y$ directions, and make average over the phase angles $\theta_{1}$ and $\theta_{2}$, then the quantity in (9) becomes

$$
\begin{aligned}
\iint d \xi_{1} d \xi_{2} \iint \frac{d \theta_{1} d \theta_{2}}{(2 \pi)^{2}} \oint \frac{i d z}{2 \pi} \operatorname{Tr} & \left\{\tilde{G} \frac{\partial \tilde{G}^{-1}}{\partial \xi_{1}} \tilde{G} \frac{\partial \tilde{G}^{-1}}{\partial \theta_{1}} \tilde{G} \frac{\partial \tilde{G}^{-1}}{\partial \xi_{2}} \tilde{G} \frac{\partial \tilde{G}^{-1}}{\partial \theta_{2}} \tilde{G}\right. \\
& \left.+\tilde{G} \frac{\partial \tilde{G}^{-1}}{\partial \theta_{2}} \tilde{G} \frac{\partial \tilde{G}^{-1}}{\partial \xi_{2}} \tilde{G} \frac{\partial \tilde{G}^{-1}}{\partial \theta_{1}} \tilde{G} \frac{\partial \tilde{G}^{-1}}{\partial \xi_{1}} \tilde{G}\right\}
\end{aligned}
$$

where we have made a gauge transformation so that the phase angle dependence in the boundary conditions goes explicitly into the Hamiltonian.

In the appendix we show that (15) can be further written as

$$
\begin{gathered}
\iiint \int \oint \frac{i d \xi_{1} d \xi_{2} d \theta_{1} d \theta_{2} d z}{480 \pi^{3}} \operatorname{Tr}\left\{\tilde{G} \frac{\partial \tilde{G}^{-1}}{\partial \xi_{1}} \tilde{G} \frac{\partial \tilde{G}^{-1}}{\partial \theta_{1}} \tilde{G} \frac{\partial \tilde{G}^{-1}}{\partial \xi_{2}} \tilde{G} \frac{\partial \tilde{G}^{-1}}{\partial \theta_{2}} \tilde{G} \frac{\partial \tilde{G}^{-1}}{\partial z}\right. \\
+119 \text { antisymmetrizing terms }\} .
\end{gathered}
$$


According to Ref.[7], such an expression takes integer values and represents the winding number for the mapping from $T^{5}$ to nonsingular operators $G$. Therefore, the density response (9) is quantized as integers.

\section{The Case With Many Body Interactions}

In this section we try to generalize the result of quantization of density response to the case with many body interactions. This can be readily done by invoking the relationship with quantized particle transport (see the next section), which has been established in the presence of many-body interactions. As we show below, such a generalization can also be done directly within the present formalism in one dimension.

Eq.(5) remains valid if we regard $G$ as the Green function for the many body Hamiltonian, regard $\hat{\rho}\left(x_{0}\right)$ as $\sum_{j=1}^{N} \delta\left(x_{j}-x_{0}\right)$, and replace $\frac{\partial V_{1}(\alpha x-\xi)}{\partial \xi} x$ by $\sum_{j=1}^{N} \frac{\partial V_{1}\left(\alpha x_{j}-\xi\right)}{\partial \xi} x_{j}$, where $j$ labels the particles. The contour integral is now surrounding the ground state energy of the many body system. Eq.(6) will also remain valid if we make similar changes on the right hand side; explicitly, the second term becomes

$$
\oint \frac{d z}{2 \pi i} \sum_{j} \operatorname{Tr}\left\{G \frac{\partial V_{1}\left(\alpha x_{j}-\xi\right)}{\partial \xi} G x_{j} \hat{\rho}\left(x_{0}\right)\right\}
$$

where the trace is over the antisymmetric many-body states only. In the coordinate representation, $G\left(x_{1}, x_{2}, \cdots, x_{N} ; x_{1}^{\prime}, x_{2}^{\prime}, \cdots, x_{N}^{\prime}\right)$, the Green function is antisymetric under permutation of each set of position variables. We may thus replace $x_{j}$ in the above equation by $\sum x_{k} / N$ under the trace operation. Then Eq.(17) becomes a total derivative in $\xi$, and can therefore be ignored. Similarly, Eq.(7) now becomes

$$
\frac{\partial \bar{\rho}\left(x_{0}\right)}{\partial \alpha}=-\frac{N}{L} \int_{0}^{2 \pi} \frac{d \theta}{2 \pi} \int d \xi \oint \frac{d z}{2 \pi i} \sum_{j} \operatorname{Tr}\left\{G \frac{\partial V_{1}\left(\alpha x_{j}-\xi\right)}{\partial \xi} G i \hbar v_{j} G\right\} .
$$

Again, because of the antisymmetry of the Green functions in particle labels, we may replace $v_{j}$ by $\sum_{k} v_{k} / N$. The final result of (8) can then be rederived, showing the quantization of density response in the presence of many body interactions.

Unfortunately, similar arguments in the two dimensional case show that the density response is given by the expression (15) or (16) divided by $N$, if we regard $G$ as the manybody Green function. This says that density response is only quantized in integer units of $1 / N$. 


\section{Density Response to a Magnetic Field}

Having studied the density response to the dilation of periodic potentials, we now consider the problem of density response to a change of a magnetic field. Associated with the magnetic field is a length, $\sqrt{\frac{\hbar}{e B}}$, the so called magnetic length, whose square gives the area occupied by one flux quantum. Our problem can therefore also be stated as how the electron density is controled by the scale of the magnetic length rather than by the unit cell length scale of a periodic potential. As we pointed out before, this is a problem closely related to the quantum Hall effect, but our treatment will be shaped in a way more parallel to the method used in the previous sections than those used by other authors. [8]

Consider a two dimensional electron gas in a uniform magnetic field $B$ in the perpendicular direction. The single electron Hamiltonian may be written as

$$
H=\frac{1}{2 m}(\mathbf{p}+\mathbf{e A})^{2}+\mathbf{V}(\mathbf{r})
$$

where $\mathbf{A}$ is the vector potential and may be taken as $\left(A_{1}, B x+A_{2}\right)$ with $A_{1}$ and $A_{2}$ being constants independent of $B$. We have no special requirement on the scalor potential $V(\mathbf{r})$. The particle density is still given by a formula like in (4) except that $\rho\left(x_{0}\right)$ should be replaced by the corresponding $2 \mathrm{D}$ quantity $\rho\left(\mathbf{r}_{0}\right)$. Since the magnetic field dependence comes only from the Green function, the differential density response to the magnetic field can be calculated using the formula $d G=-G d G^{-1} G$ as

$$
\frac{\partial \rho\left(\mathbf{r}_{0}\right)}{\partial B}=-\oint \frac{d z}{2 \pi i} \operatorname{Tr}\left\{G \frac{\partial H}{\partial A_{2}} x G \hat{\rho}\left(\mathbf{r}_{0}\right)\right\}
$$

Next, we note as before that $x G$ may be written as $G\left[G^{-1}, x\right] G+G x=\frac{i \hbar}{e} G \frac{\partial H}{\partial A_{1}} G+G x$. Equation (20) can then be written as

$$
\frac{\partial \rho\left(\mathbf{r}_{0}\right)}{\partial B}=-\frac{\hbar}{e} \oint \frac{d z}{2 \pi} \operatorname{Tr}\left\{G \frac{\partial H}{\partial A_{2}} G \frac{\partial H}{\partial A_{1}} G \hat{\rho}\left(\mathbf{r}_{0}\right)\right\}
$$

where we have dropped a term proportional to the $z$-integral of $\operatorname{Tr}\left\{G \frac{\partial H}{\partial A_{2}} G x \hat{\rho}\left(\mathbf{r}_{0}\right)\right\}$. Such a term is proportional to $x_{0}$ times the derivative of $\rho\left(\mathbf{r}_{0}\right)$ with respect to $A_{2}$, and is zero because of gauge invariance. 
As before, the z-integral goes along a contour in the complex energy plane enclosing the spectrum of filled states. The contour will be chosen everywhere away from the real axis (where the spectrum lies), except at the Fermi energy and a point below the whole spectrum. We assume that there are no extended states in the bulk at the Fermi energy. It then follows that the Green function in the coordinate representation $G\left(\mathbf{r}, \mathbf{r}^{\prime}\right)$ is essentially local, i,e., it decays exponentially at large $\left|\mathbf{r}-\mathbf{r}^{\prime}\right| .[5]$ We can thus impose periodic boundary conditions to the wave functions, making only exponentially small errors in the density response in the bulk. We can then average over $r_{0}$ on the boundaryless torus, yielding

$$
\frac{\partial \rho\left(\mathbf{r}_{0}\right)}{\partial B}=-\frac{\hbar}{e L_{1} L_{2}} \oint \frac{d z}{2 \pi} \operatorname{Tr}\left\{G \frac{\partial G^{-1}}{\partial A_{2}} G \frac{\partial G^{-1}}{\partial A_{1}} G\right\}
$$

Also upto exponentially small errors, we can make a further average over $A_{1}$ and $A_{2}$ in the intervals $\left(0, \frac{h}{e L_{1}}\right)$ and $\left(0, \frac{h}{e L_{2}}\right)$ corresponding to unit flux quanta through the holes of the torus, with the result

$$
\frac{\partial \rho\left(\mathbf{r}_{0}\right)}{\partial B}=-\frac{e}{h} \oint \frac{d z}{(2 \pi)^{2}} \iint d A_{1} d A_{2} \operatorname{Tr}\left\{G \frac{\partial G^{-1}}{\partial A_{2}} G \frac{\partial G^{-1}}{\partial A_{1}} G\right\}
$$

which is essentially the same as Eq.(1) of Ref.[6], because the above expression is antisymmetric upon interchanging $A_{1}$ and $A_{2}$, and because the $z$-derivative of $G^{-1}$ is unity. The density response to the magnetic field is therefore quantized in units of $e / h$.

As has been shown in the context of the quantum Hall effect, the above result allows a number of generalizations. First, the system does not have to be strictly two dimensional. Also, one can add a modulation to the magnetic field without changing the quantization of the density response to the uniform part of the field. Further, one can reformulate the above derivations using many-body quantities (cf. Section 4), allowing interactions between electrons. Finally, when the many-body state does not go back to itself when a flux quantum is inserted through a hole of the torus, which occurs in a fractional quantum Hall regime, one obtains fractionally quantized density response to the field.

\section{Applications}

In one dimension, quantized density response to a dilation of a periodic potential is the same as the quantized adiabatic particle transport studied by Thouless and others. $[8,5]$ When $\alpha$ is increased by $\delta \alpha$, the potential $V_{1}$ shrinks a distance of $\frac{\delta \alpha L}{2 \alpha}$ at $x= \pm L / 2$. If 
the particle transport is $t$ when the potential is shifted by a period $(1 / \alpha)$, there will be $t \alpha \delta \alpha L /(2 \alpha)=t \delta \alpha L / 2$ particles transported inward through the point $x=L / 2$, and there will be the same amount of particles transported inward through the point at $x=-L / 2$. The density increase is therefore $t \delta \alpha$. In fact, the expression (7) is identical to that for the particle transport. However, the concept of density response can be readily generalized to more than one dimension.

The quantized density response as a topological invariant has an important physical content: the system may be classified into topologically distinct situations, two situations being topologically the same if one can be continuously transformed into the other without closing the gap at the Fermi energy. Two situations should have the same integer of density response if they are topologically identical. This follows from the fact that the expression for the density response is a continuous functional of the Hamiltonian, and from the fact that the value of the density response is discrete.

One special class is for $V_{1}=$ const. or weak, where we have to assume that $V_{2}$ is such that there is always a gap at the Fermi energy. A $V_{1}$ potential is considered weak as long as it does not close the energy gap at the Fermi energy as it is turned on from zero. The integer of density response for this class is zero.

Another special class is for $V_{2}=$ const. or weak. For this class we only need to consider the case of $V_{2}=0$. The density response for $n$ filled bands is simply $n$, because there is one electron per unit cell for each filled band, discounting the spin degeneracy.

More interesting situations occur when the potential is a superposition of periodic ones. Consider the following example in one dimension:

$$
V(x)=\sum_{j} V_{j}\left(\alpha_{j} x-\xi_{j}\right)
$$

where each $V_{j}$ has period 1 in $\xi_{j}$. We assume that the gap at the Fermi energy does not close for a neighborhood of $\alpha_{j}$, and that $t_{j}$ is the integer of density response to the $j$ th potential, then

$$
\delta \bar{\rho}=\sum_{j} t_{j} \delta \alpha_{j} .
$$

Under a uniform scaling of $x \rightarrow(1+\delta \alpha) x$, we have $\delta \bar{\rho}=\bar{\rho} \delta \alpha$. On the other hand, such a scaling is equivalent to setting $\delta \alpha_{j}=\alpha_{j} \delta \alpha$, so that $\delta \bar{\rho}=\delta \alpha \sum_{j} t_{j} \alpha_{j}$, according to (25). 
Comparison of the two results yields

$$
\bar{\rho}=\sum_{j} t_{j} \alpha_{j} .
$$

A similar result has been obtained by Thouless using quantized particle transport. [9] In higher dimensions, the present theory allows us to generalize the above result to

$$
\bar{\rho}=\sum_{j} t_{j} / v_{j},
$$

where $v_{j}$ is the unit cell volume of the $j$ th potential.[10]

In two dimensions and in the presence of a magnetic field $B$, one has to add to the right hand side of (27) an extra term $\sigma e \delta B / h$, where $\sigma$ is the integer for the quantum Hall effect. Under a uniform scaling of $\delta \alpha$, we have to also set $\delta B=\delta \alpha B$, then we have

$$
\bar{\rho}=\sum_{j} t_{j} / v_{j}+\sigma e B / h .
$$

Such a result was first derived by Wannier in the study of the Harper's equation[11], and has been proved in general by Dana et al using magnetic translation group. [4] A three dimensional generalization of the above relation has been studied in Ref.[12].

There are two remaining problems to be solved. The first is to generalize the result of density response quantization to three (and higher) dimensions. It may be possible to do so within the present formalism, but the algebra involved will be quite heavy. The second is to show (or disprove) the quantization in two or more dimensions with particle particle interactions present. It is possible that fractionally quantized density response may occur for the interacting case.[13]

\section{Acknowledgment}

The author wishes to thank X. G. Wen, D. J. Thouless, Y. Avron and E. Akkermans for useful discussions, and to thank the hospitality of the Institute for Theoretical Physics at Technion, Israel, where part of this work was done. This work was supported by the Welch Foundation and by an NIST Precision Measurement Grant. 


\section{Appendix}

To simplify notations, we represent the quantity in the square brackets in (15) symbolically as

$$
\xi_{1} \theta_{1} \xi_{2} \theta_{2}+\theta_{2} \xi_{2} \theta_{1} \xi_{1}
$$

The original problem is symmetric under the exchange of $(1 \leftrightarrow 2),(\mathrm{A} 1)$ is equivalent to half of

$$
\begin{gathered}
\xi_{1} \theta_{1} \xi_{2} \theta_{2}+\theta_{2} \xi_{2} \theta_{1} \xi_{1} \\
+\xi_{2} \theta_{2} \xi_{1} \theta_{1}+\theta_{1} \xi_{1} \theta_{2} \xi_{2} .
\end{gathered}
$$

By integrating $z$ by parts, we can rewrite (A1) as

$$
\begin{aligned}
& -\theta_{1} \xi_{2} \theta_{2} \xi_{1}-\xi_{2} \theta_{2} \xi_{1} \theta_{1}-\theta_{2} \xi_{1} \theta_{1} \xi_{2} \\
& -\xi_{2} \theta_{1} \xi_{1} \theta_{2}-\theta_{1} \xi_{1} \theta_{2} \xi_{2}-\xi_{1} \theta_{2} \xi_{2} \theta_{1}
\end{aligned}
$$

Therefore, (A2) is equal to

$$
\begin{aligned}
& -\theta_{1} \xi_{2} \theta_{2} \xi_{1}-\theta_{2} \xi_{1} \theta_{1} \xi_{2} \\
& -\xi_{2} \theta_{1} \xi_{1} \theta_{2}-\xi_{1} \theta_{2} \xi_{2} \theta_{1} .
\end{aligned}
$$

It then follows that (A1) may be written as $\frac{1}{4}$ of (A2) plus (A4), i.e.

$$
\begin{aligned}
& \xi_{1} \theta_{1} \xi_{2} \theta_{2}-\xi_{1} \theta_{2} \xi_{2} \theta_{1} \\
+ & \theta_{2} \xi_{2} \theta_{1} \xi_{1}-\theta_{2} \xi_{1} \theta_{1} \xi_{2} \\
+ & \xi_{2} \theta_{2} \xi_{1} \theta_{1}-\xi_{2} \theta_{1} \xi_{1} \theta_{2} \\
+ & \theta_{1} \xi_{1} \theta_{2} \xi_{2}-\theta_{1} \xi_{2} \theta_{2} \xi_{1} .
\end{aligned}
$$

The first line of (A5) may be written as

$$
\begin{array}{r} 
\\
\tilde{G} \frac{\partial \tilde{G}^{-1}}{\partial \xi_{1}} \frac{\partial \tilde{G}}{\partial \theta_{1}} \frac{\partial \tilde{G}^{-1}}{\partial \xi_{1}} \frac{\partial \tilde{G}}{\partial \theta_{2}}-\tilde{G} \frac{\partial \tilde{G}^{-1}}{\partial \xi_{1}} \frac{\partial \tilde{G}}{\partial \theta_{2}} \frac{\partial \tilde{G}^{-1}}{\partial \xi_{1}} \frac{\partial \tilde{G}}{\partial \theta_{1}} \\
=-\frac{\partial \tilde{G}}{\partial \theta_{1}} \frac{\partial \tilde{G}^{-1}}{\partial \xi_{1}} \tilde{G} \frac{\partial \tilde{G}^{-1}}{\partial \xi_{1}} \frac{\partial \tilde{G}}{\partial \theta_{2}}+\frac{\partial \tilde{G}}{\partial \theta_{2}} \frac{\partial \tilde{G}^{-1}}{\partial \xi_{1}} \tilde{G} \frac{\partial \tilde{G}^{-1}}{\partial \xi_{1}} \frac{\partial \tilde{G}}{\partial \theta_{1}}
\end{array}
$$

where we have integrated $\theta_{1}$ by parts in the first term, and $\theta_{2}$ in the second term. Written in abstract notation, (A6) is $\theta_{2} \xi_{1} \xi_{2} \theta_{1}-\theta_{1} \xi_{1} \xi_{2} \theta_{2}$. Similar manipulations can be made on 
the remaining lines of (A5), showing that (A5) is equal to

$$
\begin{aligned}
& \theta_{2} \xi_{1} \xi_{2} \theta_{1}-\theta_{1} \xi_{1} \xi_{2} \theta_{2} \\
+ & \theta_{1} \xi_{2} \xi_{1} \theta_{2}-\theta_{2} \xi_{2} \xi_{1} \theta_{1} \\
+ & \xi_{2} \theta_{1} \theta_{2} \xi_{1}-\xi_{1} \theta_{1} \theta_{2} \xi_{2} \\
+ & \xi_{1} \theta_{2} \theta_{1} \xi_{2}-\xi_{2} \theta_{2} \theta_{1} \xi_{1} .
\end{aligned}
$$

Next, by integrating $z$ by parts, we can show that (A7) is further equal to

$$
\begin{aligned}
& \xi_{1} \xi_{2} \theta_{2} \theta_{1}-\xi_{1} \xi_{2} \theta_{1} \theta_{2} \\
+ & \xi_{2} \xi_{1} \theta_{1} \theta_{2}-\xi_{2} \xi_{1} \theta_{2} \theta_{1} \\
+ & \theta_{1} \theta_{2} \xi_{2} \xi_{1}-\theta_{1} \theta_{2} \xi_{1} \xi_{2} \\
+ & \theta_{2} \theta_{1} \xi_{1} \xi_{2}-\theta_{2} \theta_{1} \xi_{2} \xi_{1} .
\end{aligned}
$$

In summary, we may write $(\mathrm{A} 1)$ as $\frac{1}{12}$ of $(\mathrm{A} 5)+(\mathrm{A} 7)+(\mathrm{A} 8)$, namely

$$
\tilde{G} \frac{\partial \tilde{G}^{-1}}{\partial \xi_{1}} \tilde{G} \frac{\partial \tilde{G}^{-1}}{\partial \theta_{1}} \tilde{G} \frac{\partial \tilde{G}^{-1}}{\partial \xi_{2}} \tilde{G} \frac{\partial \tilde{G}^{-1}}{\partial \theta_{2}} \tilde{G}+23 \text { antisymetrizing terms, }
$$

where 'antisymmetrizing' acts on the four variables $\xi_{1}, \theta_{1}, \xi_{2}$, and $\theta_{2}$. Then, we append each term a factor of $\frac{\partial \tilde{G}^{-1}}{\partial z}=1$, and use the cyclic property of the trace, yielding the expression (16) in the text. 


\section{REFERENCES}

1. P. Streda, J. Phys. C 15, L717 (1982).

2. P. Streda, J. Phys. C 15, L1299 (1982).

3. D. J. Thouless, M. Kohmoto, M. P. Nightingale, and M. den Nijs, Phys. Rev. Lett. 49, 405 (1982).

4. I. Dana, Y. Avron, and J. Zak, J. Phys. C 18, L679 (1985); J. E. Avron and L. G. Yaffe, Phys. Rev. Lett. 56, 2084 (1986).

5. Q. Niu and D. J. Thouless, J. Phys. A 17, 2453 (1984).

6. H. Aoki and T. Ando, Phys. Rev. Lett. 57, 3093 (1986).

7. E. Witten, Nucl. Phys. B 223, 422 (1983); R. Bott and R. Seeley, Commun. Math. Phys. 62, 235 (1978). The base manifolds considered in these references are spheres instead of tori, but the quantization of an expression like Eq.(16) is valid independent of the topology of the base manifold.

8. J. E. Avron, R. Seiler, and B. Simon, Phys. Rev. Lett. 65, 2185 (1990). J. Bellisard, In proceedings of the Bad-Schandau Conference on Localization, edited by W. Weller and P. Zieshe (Teubner, Leipzig, 1988); J. Xia, Comm. Math. Phys. 119, 29-50 (1988).

9. D. J. Thouless, Phys. Rev. B 27, 6083 (1983); Q. Niu, Phys. Rev. B34 (1986) 5093. Also see Ref.[5].

10. R. Johnson and J. Moser, Commun. Math. Phys. 84, 403 (1982).

11. G. H. Wannier, Phys. Status Solidi 88, 757 (1978)

12. G. Montambaux and M. Kohmoto, Phys. Rev. B 41, 11417 (1990); M. Kohmoto, B. I. Halperin, and Y-S. Wu, Phys. Rev. B 45, 13488 (1992).

13. See Section 5 in the context of quantum Hall effect, and see Q. Niu, Mod. Phys. Lett. B5, (1991) 923, in the context of adiabatic particle transport. 